\title{
Developing a synthetic psychosocial stress measure and harmonizing CVD-risk data: a way forward to GxE meta- and mega-analyses
}

\author{
Abanish Singh ${ }^{1,2,3^{*}}$ (D), Michael A. Babyak ${ }^{1,2}$, Beverly H. Brummett ${ }^{1,2}$, William E. Kraus ${ }^{3,4}$, llene C. Siegler ${ }^{1,2}$, \\ Elizabeth R. Hauser ${ }^{3,5}$ and Redford B. Williams ${ }^{1,2}$
}

\begin{abstract}
Objectives: Among many challenges in cardiovascular disease (CVD) risk prediction are interactions of genes with stress, race, and/or sex and developing robust estimates of these interactions. Improved power with larger sample size contributed by the accumulation of epidemiological data could be helpful, but integration of these datasets is difficult due the absence of standardized phenotypic measures. In this paper, we describe the details of our undertaking to harmonize a dozen datasets and provide a detailed account of a number of decisions made in the process.

Results: We harmonized candidate genetic variants and CVD-risk variables related to demography, adiposity, hypertension, lipodystrophy, hypertriglyceridemia, hyperglycemia, depressive symptom, and chronic psychosocial stress from a dozen studies. Using our synthetic stress algorithm, we constructed a synthetic chronic psychosocial stress measure in nine out of twelve studies where a formal self-rated stress measure was not available. The mega-analytic partial correlation between the stress measure and depressive symptoms while controlling for the effect of study variable in the combined dataset was significant $(R h O=0.27, p<0.0001)$. This evidence of the validity and the detailed account of our data harmonization approaches demonstrated that it is possible to overcome the inconsistencies in the collection and measurement of human health risk variables.
\end{abstract}

Keywords: Data harmonization, GxE interaction, CVD-risk, Mega-analysis, Synthetic psychosocial stress, Depressive symptoms, Correlation

\section{Introduction}

Psychosocial stress, defined as aversive or demanding environmental conditions that exceed the resources of an organism, has often been implicated in the genesis of cardiovascular disease (CVD) and CVD-risk factors [1, 2]. Stress also may play a significant role in modifying the impact of genetic factors on CVD-risk [3, 4]. A better understanding of how genes interact with stress to contribute to CVD pathways might be gained by developing robust estimates of gene-by-stress interactions across the disease pathways in the context of genetic variations and demographic differences.

\footnotetext{
*Correspondence: abanish.singh@duke.edu

${ }^{1}$ Behavioral Medicine Research Center, Duke University School of Medicine, Durham, NC, USA

Full list of author information is available at the end of the article
}

Detecting and generalizing statistical interaction typically requires considerably larger sample sizes than needed for statistical main effects [5]. Moreover, some interactions are observable only in limited situations and may not be broadly generalizable [6]. One approach to overcoming these challenges is to exploit the large accumulation of epidemiological data, thereby increasing sample size and statistical power. These data can be used to conduct a conventional meta-analysis, a potential new standard of original research [7], in which an aggregate estimate is generated using summary statistics from individual new studies or already reported in the literature [8]. Alternatively, the individual-level data can be combined into a single harmonized dataset upon which new analyses are carried out. This latter approach is often referred to as mega-analysis [8]. 
Studies with inconsistent measurements, protocols, and methods may lead to inconsistent conclusions. Moreover, the integration of measures across studies with heterogeneous measurement protocols, units and coding can be a significant challenge. This challenge has resulted in several data harmonization efforts [9-11], but these have focused mostly on the design and standardization of measures for use in future studies. A more significant challenge is the circumstance in which there may not be an explicit measure of the phenotype of interest in existing studies. In pursuing our work on psychosocial stress, we were immediately confronted by the absence of an explicit measure of psychosocial stress in many studies. Thus, an important undertaking in our previous work was to develop an algorithm for constructing a valid measure of psychosocial stress from extant datasets with no explicit stress measure [4]. We refer to this as a "synthetic" measure of psychosocial stress to distinguish it from formal self-rated measures developed specifically to assess stress. Our synthetic stress algorithm is based on the items of the formal, self-rated measure of chronic psychosocial stressors known as "chronic burden" in the Multi-Ethnic Study of Atherosclerosis (MESA) [12]. The MESA chronic burden measure, as well as similar indicators of stress, are associated with a range of CVD-risk factors $[3,13-16]$.

In the present paper, we provide a detailed illustration of a number of decisions made in the process of creating the synthetic stress measure, the harmonization of inconsistency among CVD-risk variables, and subsequent combining the harmonized data from a dozen different studies into a single dataset. We also provide a mega-analytic estimate of association between stress and depressive symptoms. The harmonized data matrix also included single nucleotide polymorphisms (SNPs) EBF1 rs4704963, 5HTR2C rs6318, and BDNF rs6265, which we found associated with CVD-risk factors in the presence of stress in our earlier work $[3,17,18]$. These efforts will allow us to develop robust estimates of gene-by-stress interactions.

\section{Main text}

\section{Methods and material}

\section{Data sources}

We used a dozen (six dbGaP and six Duke) datasets in this data harmonization study. The dbGaP public-access datasets were from the Women's Health Initiative (WHI) Study [19]; Coronary Artery Risk Development in Young Adults Study (CARDIA) [20]; Atherosclerosis Risk in Communities Study (ARIC) [21]; Framingham Offspring Cohort [22]; Multi-Ethnic Study of Atherosclerosis (MESA) [23]; and Jackson Heart Study (JHS) [24]. The Duke datasets were from the Community Health and Stress Evaluation (CHASE) Study [25]; Duke Family Heart Study (DFHS) [26]; Duke Caregiver Study (DCS) [27]; and three cohorts for Studies of a Targeted Risk Reduction Intervention through Defined Exercise (STRRIDE), i.e., STRRIDE I [28], STRRIDE-Aerobic Training/Resistance Training (AT/RT) [29], and STRRIDE Pre-Diabetes (PD) [30] studies. A brief description of the contributing studies is provided in the Additional file 1 .

\section{Building a synthetic stress measure}

Using the algorithm described in [4], we constructed our synthetic stress measure in four out of six dbGaP datasets and five out of six Duke datasets where a self-rated formal stress measure was not available. The MESA and JHS datasets included a self-rated stress measure. In the absence of items that specifically query about stress, the algorithm uses proxy indicators of the domains used in the MESA chronic burden measure [12]: financial strains, relationship or marital problems, difficulties with job or ability to work, serious health problems of spouse or someone close, and one's own serious health problems. The steps of our algorithm [4] included searching the most suitable proxy item for as many of the five components as possible, scoring them as 0 or 1 using the proxy item, and creating the synthetic Singh et al. [4] chronic stress ordinal variable by summing all available binary components. Our analysis in previous work suggested that a synthetic variable developed using incomplete set of two (worst case), three, or four proxy items could still be useful, when all five proxy items were not available.

Pseudocodes We provide a description and pseudocodes for the construction of synthetic stress measure in all studies in the Additional file 1.

\section{Validation of synthetic stress measure}

Assuming that the samples under study were at least broadly similar culturally, we evaluated the distributions of the synthetic stress measure in each dataset and compared them with available self-rated measures, and expected the shape of distributions to be reasonably similar across studies. We provided additional support for the validity of the synthetic stress measure by evaluating its well-known association (Spearman correlation) with a measure of depressive symptoms.

Mega-analysis We also estimated a partial correlation of stress and depressive symptoms in harmonized, combined data whilst controlling for the effect of study dummy variables.

\section{Additional steps in data harmonization}

Harmonizing variability in units and coding For phenotype measures that were presented in different units 
across the studies, we used accepted conversion factors (Additional file 1: Table S1) to create a corresponding single unified variable. The inconsistent codings for sex and race were also reconciled. Ordinal measures of a phenotype that differed in terms of the number of possible responses (e.g., chronic stress, depressive symptoms) were converted to $z$-scores $(\mathrm{SD}=1$, mean $=0)$ within each study.

Accounting for data sources In order to facilitate the mega-analysis using combined multiple datasets, we created vectors of dummy indicators for each study. These variables enable adjustment for study origin and as possible effect modifiers in fixed effects models. More details on the choice of dummy variable over random effects coding are provided in Additional file 1.

Dealing with outliers Extreme outlying values can unduly influence statistical estimates of association or central tendency and are typically excluded or trimmed to less extreme values. One potential challenge in this regard is that it is not always possible to determine whether outliers are the actual measured values or the result of errors. More details on outlier detection and removal are provided in Additional file 1.

Summary statistics Finally, we evaluated summary statistics and distributions of harmonized data variables in order to evaluate consistency in the harmonized measurements and differences across the study cohorts.

\section{Genetic data: identifying proxy SNPS}

We harmonized the candidate SNPs of interest (rs4704963, rs6318, and rs6265, as reviewed above) across all datasets. We identified proxy SNPs for a missing SNP using two criteria (1) a high score of the proxy SNP with the SNP of interest (Linkage Disequilibrium $\left.R^{2} \geq 0.95\right)$ and (2) availability of same proxy SNP in each dataset. The SNP data for each study was subjected to a standard quality control before selecting the candidate SNPs (Additional file 1).

\section{Results}

The distribution of the chronic psychosocial stress $z$-scores are presented in Fig. 1 . The synthetic stress variable appears for all datasets, with the exception of MESA, which used the aforementioned chronic burden measure, and JHS, which, in addition to the five domains, also assessed stress due to legal problems, racism/discrimination, and neighborhood characteristics. The similarity in shapes of z-scores distributions (i.e., flat, skewed toward the right; kurtosis $=2.19-7.92$, skewness $=0.20-2.34$ ) adds additional support to our contention that the synthetic stress was assessing a similar underlying construct in different studies.
The Spearman correlations of the synthetic measures (Table 1) for all datasets except CARDIA $(R h o=0.07)$ were reasonably strong $(R h o=0.20-0.57)$, significantly different from zero $(p<0.001)$, and similar in magnitude to those observed for the self-rated measures (i.e., MESA and JHS). Some of the possible reasons for the weak correlation in CARDIA may be due to the facts that the available CES-D depression measure was assessed in a later exam that followed baseline and that it was the youngest cohort (mean age 24.97 years). Controlling for the effect of study variable, mega-analytic partial correlation between stress and depression in combined dataset was significant $(R h o=0.27, p<0.0001)$. As expected, the significant correlations between the measures of synthetic stress and depressive symptoms in all datasets except one further supports the validity of our method for the construction of synthetic stress measures in datasets that lacked a self-rated measure.

Although the units for blood pressure, BMI, and age were consistent across the datasets, studies differed in the units of other measures such as fasting glucose, insulin, and lipids (Additional file 1: Table S2 Panel A and B). The codings for sex and race were also inconsistent across the datasets. Finally, the CES-D depression measure was also scored differently in Framingham Heart Study (range 0-0.85) as compared to other datasets and a shortened version of the CES-D was used in WHI, which were converted to $\mathrm{z}$-scores. While the uniformity in the summary statistics of harmonized variables (Additional file 1: Table S3) support the tenability of the harmonization process, they also demonstrate the underlying differences in each cohort in terms of age and CVD-risk factors. The distribution plots of each CVD-risk variable for each datasets (Fig. 2a, b) provide a comparison of harmonized measurements across all the datasets and document the consistency of our harmonization approaches.

The harmonization of three SNPs, which moderated the influence of stress on CVD-risk endophenotypes in our prior research, resulted in proxy SNPs in the place of missing SNPs in dataset(s) with perfect LD score $\left(R^{2}=1.0\right)$. The minor allele frequency (MAF) differences among the Whites and Blacks suggested that race-stratified analysis of genetic association might be preferred for these SNPs (Additional file 1: Table S4).

\section{Discussion}

In our previous work [4], we provided an algorithm to construct synthetic stress measure and a systematic comparison of a synthetic and self-rated measure with evidences for unidimensionality using the MESA dataset. In the present work, we describe the details of our undertaking to harmonize twelve datasets and we provide the set of proxy indicators and pseudocodes for 


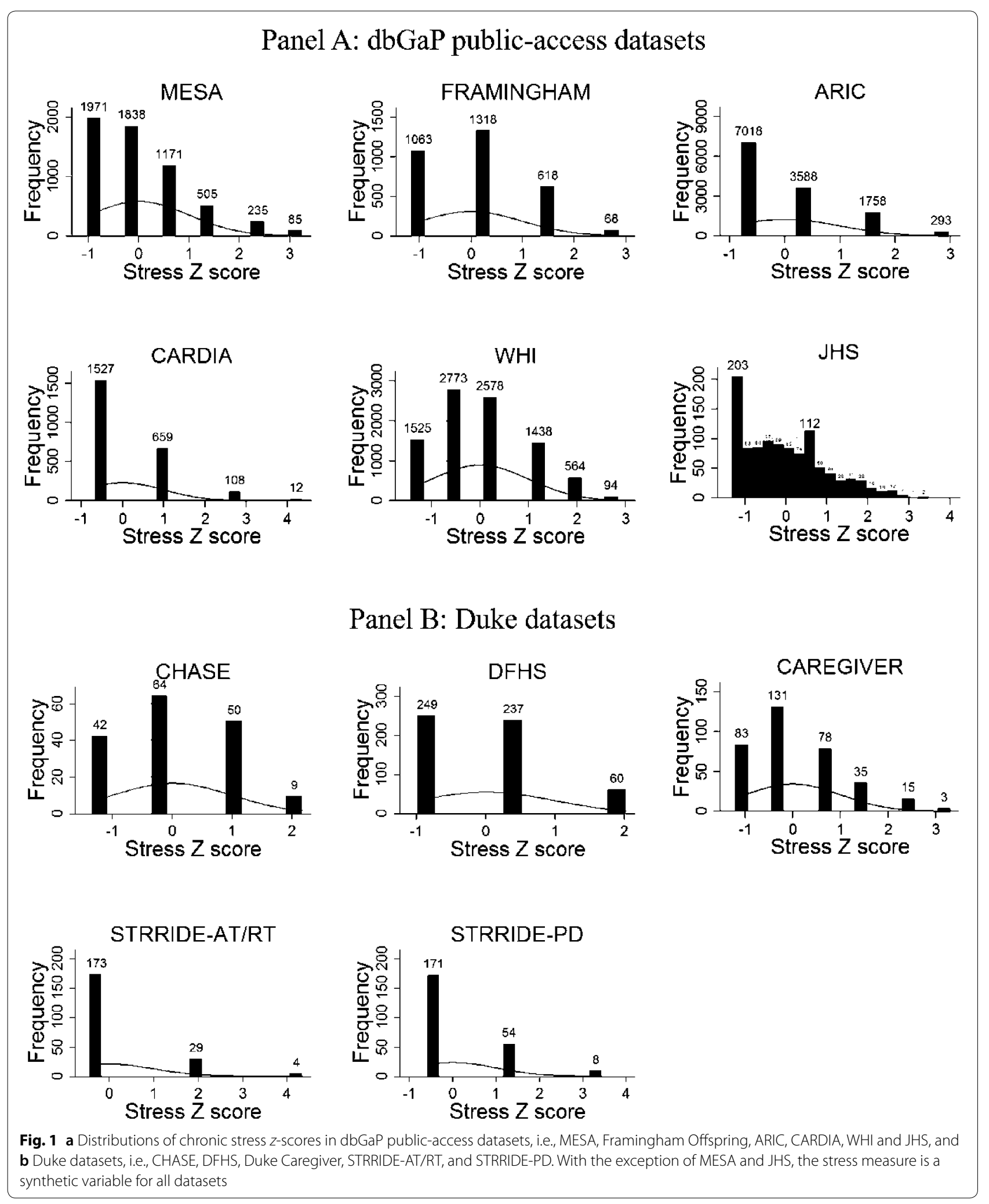


Table 1 Spearman's correlation of synthetic and self-rated stress measures with CES-D depression measure

\begin{tabular}{|c|c|c|c|c|}
\hline Dataset & Stress measure & Depression measure & Rho & P-value \\
\hline MESA & Self-rated & $\begin{array}{l}\text { Center for Epidemiological Studies-Depres- } \\
\text { sion Scale (CES-D) }\end{array}$ & 0.35 & $<0.0001$ \\
\hline Framingham Offspring Cohort & Synthetic & CES-D & 0.23 & $<0.0001$ \\
\hline CARDIA & Synthetic & CES-D & 0.07 & $<0.001$ \\
\hline ARIC & Synthetic & Maastricht Vital Exhaustion Score & 0.28 & $<0.0001$ \\
\hline WHI & Synthetic & Shortened CES-D & 0.2 & $<0.0001$ \\
\hline JHS & Self-rated & CES-D & 0.32 & $<0.0001$ \\
\hline CHASE & Synthetic & Beck Depression Inventory (BDI) & 0.31 & $<0.001$ \\
\hline DFHS & Synthetic & CES-D & 0.25 & $<0.0001$ \\
\hline Caregiver & Synthetic & CES-D & 0.42 & $<0.0001$ \\
\hline STRRIDE-AT/RT & Synthetic & Self-rated & 0.4 & $<0.0001$ \\
\hline STRRIDE-PD & Synthetic & Self-rated & 0.54 & $<0.0001$ \\
\hline Combined estimate $^{a}$ & & & 0.27 & $<0.0001$ \\
\hline
\end{tabular}

a Combined estimate derived from mega-analytic partial correlation between standardized (z-scores) stress and depression measures whilst controlling for the effect of study variable in combined dataset

constructing synthetic stress in nine out of twelve studies in hope that it will help the scientific community in further work. Two studies (MESA, JHS) had a formal self-rated stress measure and one study (STRRIDE-I) did not have any proxy indicator for synthetic measure. The construction of a synthetic stress measure in datasets that did not have a self-rated formal stress measure is a key innovation in the current study. The broad domains of psychosocial stress that we have used in our synthetic stress construction algorithm [4] have been frequently used as part of formal stress measures $[12,31]$. Thus, our synthetic stress measure is consistent with that of others using similar stress domains, which are apparently sufficient to capture life stress, even when not all present [4]. Past research has found one or more of these domains to be associated with a number of CVD-risk factors, such as, glucose metabolism [32], blood pressure [33], mortality [34], cortisol [35], and depressive symptoms [36]. Another important aspect of our work was to obtain insights from large sample resulting from combining the datasets. Summarizing results over multiple studies, either through conventional meta-analysis, or as in our case using mega-analysis (Table 1 ), is thought to produce a more robust estimate of the associations under study, and potentially more generalizable insights [37]. We provide additional discussion in the Additional file 1.

\section{Conclusions}

We illustrated our method used to construct a synthetic stress measure with evidences of its validity. Our work provides ways by which to harmonize and operationalize the existing data and overcome the inconsistencies in the collection and measurement of human health risk variables that we hope will complement and support other ongoing efforts to standardize measurements in new studies. This works also provides the opportunity of future work to perform more robust and informative mega-analytic tests of our prior findings on gene-bystress interactions modifying expression of endophenotypes in CVD pathways.

\section{Limitations}

We have so far chosen not to impute for missing variables and indicators in our approach. This choice is supported by our prior work [4] showing that the stress scores which include less than the full set of indicators still behave similarly in terms of associations with other phenotypes, such as depressive symptoms. A formal measure designed explicitly to assess stress would

(See figure on next page.)

Fig. 2 a Distributions of harmonized phenotypes in dbGaP public-access datasets. Each notched box plot shows the distribution (i.e., five point summary statistics, outliers, and notches based on the median $\pm 1.58 * \mathrm{IQR} / \mathrm{sqrt}(\mathrm{n})$ ) of one variable in the six dbGaP studies, i.e., ARIC, CARDIA, FRAMINGHAM, JACKSON HEART, MESA, and WHI; and b six Duke studies, i.e., CAREGIVER, CHASE, DFHS, STRRIDE-1, STRRIDE-AT/RT, and STRRIDE-PD. The scales for fasting glucose, insulin, $\mathrm{HbA1C}$, and triglyceride were log transformed, and the standardized depression measure was square root transformed 


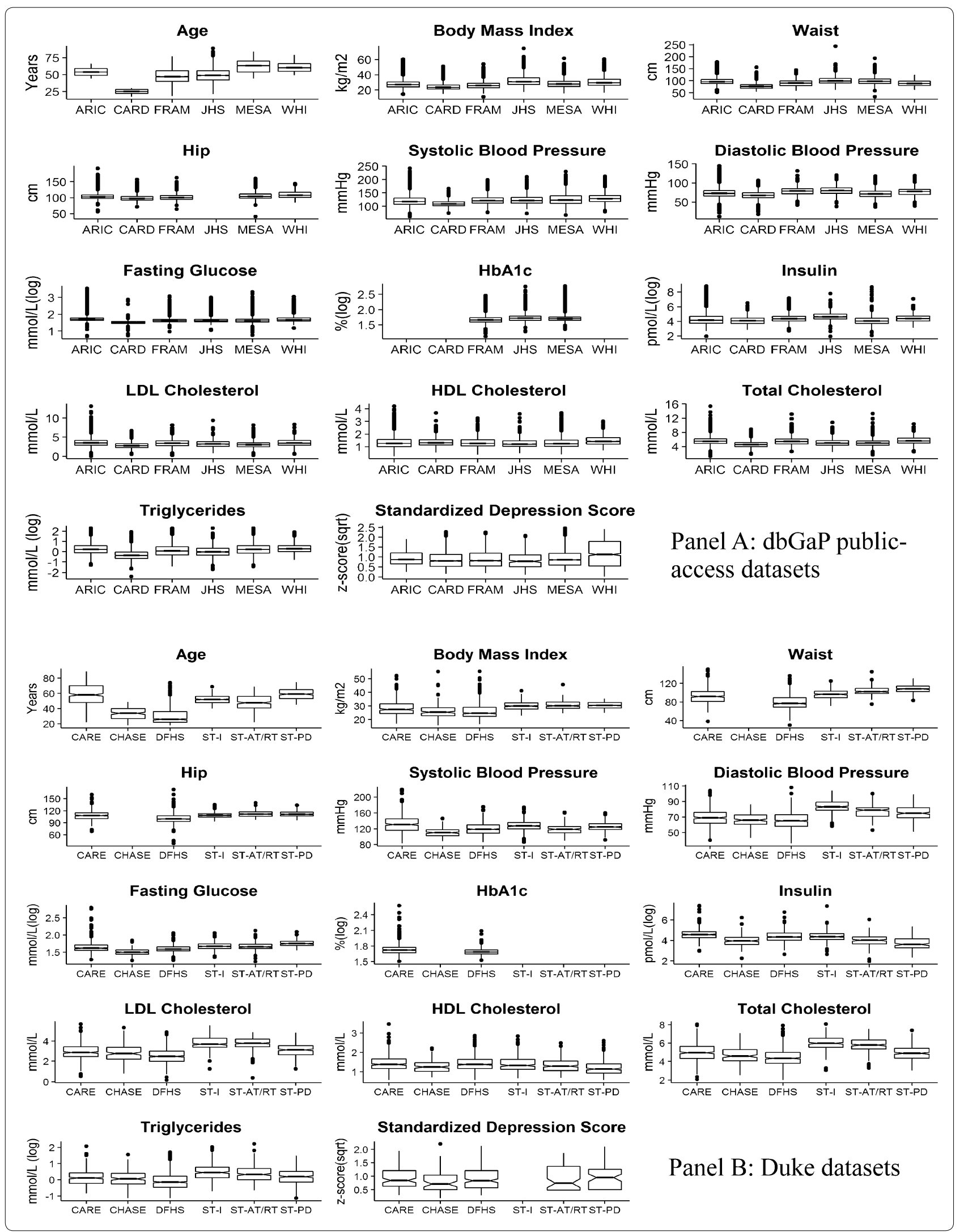


generally be the most desirable choice to use in investigations; however, when the formal measure is not available, the five-component synthetic stress measure appears to serve as an acceptable utilitarian solution.

\section{Additional file}

Additional file 1. The additional file provides more details on the data sources of contributing studies; proxy indicators and pseudocodes for synthetic stress measure; SNP quality control; coding for studies from multiple sources; outlier detection and removal; and additional discussion on the need for data harmonization, harmonization steps and alternative approaches, and insights from large sample resulting from combining the datasets

\section{Abbreviations}

CVD: cardiovascular disease; GxE: gene-by-environment interaction; SNP: single nucleotide polymorphism; EBF1: early B-cell factor 1; BDNF: brain-derived neurotrophic factor; 5HTR2C: 5-hydroxytryptamine receptor $2 \mathrm{C}$; dbGaP: database of genotypes and phenotypes; WHI: Women's Health Initiative Study; CARDIA: Coronary Artery Risk Development in Young Adults Study; MESA: Multi-Ethnic Study of Atherosclerosis; JHS: Jackson Heart Study; CHASE: Community Health and Stress Evaluation Study; DFHS: Duke Family Heart Study; DCS: Duke Caregiver Study; STRRIDE I: Study of a Targeted Risk Reduction Intervention through Defined Exercise (cohort I); STRRIDE-AT/RT: Study of a Targeted Risk Reduction Intervention through Defined Exercise-Aerobic Training/Resistance Training; STRRIDE-PD: Study of a Targeted Risk Reduction Intervention through Defined Exercise-Pre-Diabetes; BDI: Beck Depression Inventory; CES-D: Center for Epidemiological Studies-Depression Scale.

\section{Authors' contributions}

AS participated in the study design, conceived synthetic stress algorithm, obtained and harmonized public-access datasets, created synthetic stress in all datasets, performed statistical analysis, and drafted the manuscript. MAB participated in the study design, performed harmonization of Duke datasets, and reviewed and edited the manuscript. BHB participated in the study design, harmonized Duke datasets, and reviewed the manuscript. WEK participated in the study design and reviewed the manuscript. ICS participated in the study design and reviewed the manuscript. ERH participated in the study design and reviewed the manuscript. RBW supervised the study, participated in the study design, and reviewed and edited the manuscript. All authors read and approved the final manuscript.

\section{Author details \\ 1 Behavioral Medicine Research Center, Duke University School of Medicine, Durham, NC, USA. ${ }^{2}$ Department of Psychiatry and Behavioral Sciences, Duke University School of Medicine, Durham, NC, USA. ${ }^{3}$ Duke Molecular Physiology Institute, Duke University School of Medicine, Durham, NC, USA. ${ }^{4}$ Department of Medicine, Duke University School of Medicine, Durham, NC, USA. ${ }^{5}$ Depart- ment of Biostatistics and Bioinformatics, Duke University School of Medicine, Durham, NC, USA.}

\section{Acknowledgements}

The public-access datasets (MESA, Framingham, CARDIA, ARIC, WHI, and JHS) were obtained from dbGaP/database of Genotypes and Phenotypes/National Center for Biotechnology Information, National Library of Medicine (NCBI/ NLM)/https://www.ncbi.nlm.nih.gov/gap [38] through authorized/controlled data access under standard user agreement. The Duke datasets (CHASE, DFHS, CAREGIVER, and STRRIDE) were obtained from Duke studies. We thank the investigators, staff, and participants of the dbGaP and Duke studies for their valuable contributions.

\section{Competing interests}

Redford Williams is a founder and major stockholder of Williams LifeSkills, Inc. and holds a patent on the use of the 5HTTLPR L allele as a marker of stress-related CVD. Other authors have no competing interests with respect to the work.

\section{Availability of data and materials}

The public-access datasets used in this study can be obtained from the dbGaP/database of Genotypes and Phenotypes/ National Center for Biotechnology Information, National Library of Medicine (NCBI/NLM)/https://www. ncbi.nlm.nih.gov/gap through an authorized access approved by NIH Data Access Committee. The Duke datasets are however available for collaborative use upon reasonable request of collaborations with authors on permission of respective Study Committee and Duke IRB.

\section{Consent for publication}

Not applicable.

\section{Ethics approval and consent to participate}

Our study involves a secondary analysis on previously collected and anonymized human samples data and it does not report any individual participant's data or health related outcome. This secondary analysis study is approved by Duke Institutional Review Board (IRB) protocol number Pro00070669.

\section{Funding}

This work was supported by NIH/NHLBI grant P01HL036587 (Williams).

\section{Publisher's Note}

Springer Nature remains neutral with regard to jurisdictional claims in published maps and institutional affiliations.

Received: 23 March 2018 Accepted: 12 July 2018

Published online: 24 July 2018

\section{References}

1. Williams RB. Psychosocial and biobehavioral factors and their interplay in coronary heart disease. Annu Rev Clin Psychol. 2008:4(1):349-65.

2. Rosengren A, Hawken S, Ônpuu S, Sliwa K, Zubaid M, Almahmeed WA, et al. Association of psychosocial risk factors with risk of acute myocardial infarction in 11119 cases and 13648 controls from 52 countries (the INTERHEART study): case-control study. Lancet. 2004;364(9438):953-62.

3. Singh A, Babyak MA, Nolan DK, Brummett BH, Jiang R, Siegler IC, et al. Gene by stress genome-wide interaction analysis and path analysis identify EBF1 as a cardiovascular and metabolic risk gene. Eur J Hum Genet. 2015;23(6):854-62.

4. Singh A, Babyak MA, Brummett BH, Jiang R, Watkins LL, Barefoot JC, et al. Computing a synthetic chronic psychosocial stress measurement in multiple datasets and its application in the replication of $G \times E$ Interactions of the EBF1 Gene. Genet Epidemiol. 2015;39(6):489-97.

5. Brookes ST, Whitely E, Egger M, Smith GD, Mulheran PA, Peters TJ. Subgroup analyses in randomized trials: risks of subgroup-specific analyses;: power and sample size for the interaction test. J Clin Epidemiol. 2004:57(3):229-36.

6. Culverhouse RC, Saccone NL, Horton AC, Ma Y, Anstey KJ, Banaschewski T, et al. Collaborative meta-analysis finds no evidence of a strong interaction between stress and 5-HTTLPR genotype contributing to the development of depression. Mol Psychiatry. 2018;23:133-42.

7. Ioannidis JA. Meta-analyses can be credible and useful: a new standard. JAMA Psychiatry. 2017;74(4):311-2.

8. Sung YJ, Schwander K, Arnett DK, Kardia SLR, Rankinen T, Bouchard C, et al. An empirical comparison of meta-analysis and mega-analysis of individual participant data for identifying gene-environment interactions. Genet Epidemiol. 2014;38(4):369-78.

9. Fortier I, Doiron D, Little J, Ferretti V, L'Heureux F, Stolk RP, et al. Is rigorous retrospective harmonization possible? Application of the DataSHaPER approach across 53 large studies. Int J Epidemiol. 2011;40(5):1314-28.

10. Hamilton CM, Strader LC, Pratt JG, Maiese D, Hendershot T, Kwok RK, et al. The PhenX Toolkit: get the most from your measures. Am J Epidemiol. 2011;174(3):253-60 
11. Jensen MA, Ferretti V, Grossman RL, Staudt LM. The NCI Genomic Data Commons as an engine for precision medicine. Blood. 2017;130(4):453.

12. Shivpuri S, Gallo LC, Crouse JR, Allison MA. The association between chronic stress type and C-reactive protein in the multi-ethnic study of atherosclerosis (MESA): does gender make a difference? J Behav Med. 2012;35(1):74-85.

13. Troxel WM, Matthews KA, Bromberger JT, Sutton-Tyrrell K. Chronic stress burden, discrimination, and subclinical carotid artery disease in African American and Caucasian women. Health Psychol. 2003;22(3):300-9.

14. Egido JA, Castillo O, Roig B, Sanz I, Herrero MR, Garay MT, et al. Is psychophysical stress a risk factor for stroke? A case-control study. J Neurol Neurosurg Psychiatry. 2012;83(11):1104-10.

15. Bergmann N, Gyntelberg F, Faber J. The appraisal of chronic stress and the development of the metabolic syndrome: a systematic review of prospective cohort studies. Endocr Connect. 2014;3(2):R55-80.

16. Guarneri MG, Nastri L, Assennato P, Li Puma A, Landi A, Bonanno B, et al. Heart ischemia and psychosomatics: the role of stressful events and lifestyles. Monaldi Arch Chest Dis. 2009;72(2):77-83.

17. Brummett BH, Babyak MA, Jiang R, Shah SH, Becker RC, Haynes C, et al. A functional polymorphism in the 5 HTR2C gene associated with stress responses also predicts incident cardiovascular events. PLOS ONE. 2013;8(12):e82781.

18. Jiang R, Babyak MA, Brummett BH, Siegler IC, Kuhn CM, Williams RB. Brainderived neurotrophic factor (BDNF) Val66Met polymorphism interacts with gender to influence cortisol responses to mental stress. Psychoneuroendocrinology. 2017;79(Supplement C):13-9.

19. The WHI Study Group. Design of the Women's Health Initiative clinical trial and observational study. Control Clin Trials. 1998;19(1):61-109.

20. Friedman GD, Cutter GR, Donahue RP, Hughes GH, Hulley SB, Jacobs DR, et al. Cardia: study design, recruitment, and some characteristics of the examined subjects. J Clin Epidemiol. 1988:41(11):1105-16.

21. The ARIC Investigators. The atherosclerosis risk in communities (ARIC) study: design and objectives. Am J Epidemiol. 1989;129(4):687-702.

22. Feinleib M, Kannel WB, Garrison RJ, McNamara PM, Castelli WP. The framingham offspring study. Design and preliminary data. Prev Med. 1975:4(4):518-25.

23. Bild DE, Bluemke DA, Burke GL, Detrano R, Diez Roux AV, Folsom AR, et al. Multi-ethnic study of atherosclerosis: objectives and design. Am J Epidemiol. 2002;156(9):871-81.

24. Sempos CT, Bild DE, Manolio TA. Overview of the Jackson Heart Study: a study of cardiovascular diseases in African American Men and Women. Am J Med Sci. 1999;317(3):142-6.

25. Burroughs AR, Visscher WA, Haney TL, Efland JR, Barefoot JC, Williams RB, et al. Community recruitment process by race, gender, and SES gradient: lessons learned from the Community Health and Stress Evaluation (CHASE) Study experience. J Community Health. 2003;28(6):421-37.
26. Brummett BH, Boyle SH, Ortel TL, Becker RC, Siegler IC, Williams RB. Associations of depressive symptoms, trait hostility, and gender with C-reactive protein and interleukin-6 response following emotion recall. Psychosom Med. 2010;72(4):333-9.

27. Siegler IC, Brummett BH, Williams RB, Haney TL, Dilworth-Anderson P. Caregiving, residence, race, and depressive symptoms. Aging Mental Health. 2010;14(7):771-8.

28. Slentz CA, Aiken LB, Houmard JA, Bales CW, Johnson JL, Tanner CJ, et al. Inactivity, exercise, and visceral fat. STRRIDE: a randomized, controlled study of exercise intensity and amount. J Appl Physiol. 2005;99(4):1613-8.

29. Slentz CA, Bateman LA, Willis LH, Shields AT, Tanner CJ, Piner LW, et al. Effects of aerobic vs. resistance training on visceral and liver fat stores, liver enzymes, and insulin resistance by HOMA in overweight adults from STRRIDE AT/RT. Am J Physiol Endocrinol Metab. 2011;301(5):E1033.

30. Slentz CA, Bateman LA, Willis LH, Granville EO, Piner LW, Samsa GP, et al. Effects of exercise training alone vs a combined exercise and nutritional lifestyle intervention on glucose homeostasis in prediabetic individuals: a randomised controlled trial. Diabetologia. 2016;59(10):2088-98.

31. Johnson DA, Lisabeth L, Lewis TT, Sims M, Hickson DA, Samdarshi T, et al. The contribution of psychosocial stressors to sleep among African Americans in the Jackson Heart Study. Sleep. 2016;39(7):1411-9.

32. Brummett BH, Siegler IC, Rohe WM, Barefoot JC, Vitaliano PP, Surwit RS, et al. Neighborhood characteristics moderate effects of caregiving on glucose functioning. Psychosom Med. 2005;67(5):752-8.

33. Brummett BH, Babyak MA, Siegler IC, Shanahan M, Harris KM, Elder GH, et al. Systolic blood pressure, socioeconomic status, and biobehavioral risk factors in a nationally representative U.S young adult sample. Hypertension. 2011;58(2):161-6.

34. Bosworth HB, Siegler IC, Brummett BH, Barefoot JC, Williams RB, ClappChanning NE, et al. The association between self-rated health and mortality in a well-characterized sample of coronary artery disease patients. Med Care. 1999;37(12):1226-36.

35. Luecken LJ, Suarez EC, Kuhn CM, Barefoot JC, Blumenthal JA, Siegler IC, et al. Stress in employed women: impact of marital status and children at home on neurohormone output and home strain. Psychosom Med. 1997:59(4):352-9.

36. Brummett BH, Babyak MA, Williams RB, Harris KM, Jiang R, Kraus WE, et al. A putatively functional polymorphism in the HTR2C gene is associated with depressive symptoms in white females reporting significant life stress. PLOS ONE. 2014;9(12):e114451.

37. loannidis JPA, Rosenberg PS, Goedert JJ, O'Brien TR. Commentary: metaanalysis of individual participants' data in genetic epidemiology. Am J Epidemiol. 2002;156(3):204-10.

38. Mailman MD, Feolo M, Jin Y, Kimura M, Tryka K, Bagoutdinov R, et al. The NCBI dbGaP database of genotypes and phenotypes. Nat Genet. 2007:39:1181.

\footnotetext{
Ready to submit your research? Choose BMC and benefit from:

- fast, convenient online submission

- thorough peer review by experienced researchers in your field

- rapid publication on acceptance

- support for research data, including large and complex data types

- gold Open Access which fosters wider collaboration and increased citations

- maximum visibility for your research: over 100M website views per year
}

At BMC, research is always in progress.

Learn more biomedcentral.com/submissions 